\title{
Simulation of the Optimized Structure of a Laterally Coupled Distributed Feedback (LC-DFB) Semiconductor Laser Above Threshold
}

\author{
Mahmood Seifouri \\ Shahid Rajaee Teacher Training University, \\ Faculty of ECE, \\ Lavizan, Tehran, Iran \\ mahmood.seifouri@srttu.edu
}

\author{
Ata Faraji \\ Ministry of Education \\ Education Organization of Kurdistan \\ Bijar, Iran \\ faraji_elec@yahoo.com
}

\begin{abstract}
In this paper, the laterally coupled distributed feedback semiconductor laser is studied. In the simulations performed, variations of structural parameters such as the grating amplitude $a$, the ridge width $W$, the thickness of the active region $d$, and other structural properties are considered. It is concluded that for certain values of structural parameters, the laser maintains the highest output power, the lowest distortion Bragg frequency $\delta L$ and the smallest changes in the wavelength $\lambda$. Above threshold, output power more than $40 \mathrm{~mW}$ and SMSR values greater than $50 \mathrm{~dB}$ were achieved.
\end{abstract}

Keywords-Laterally coupled distributed feedback laser; rate equation; coupled wave equation; transfer matrix method

\section{INTRODUCTION}

The semiconductor DFB lasers, due to their low threshold current, narrow line width and single mode capability, are considered as the optical source in communications systems [1]. Conventional DFB lasers with coupling refractive index with end facets coated with anti reflection (AR), are not singlemoded and have two symmetrical modes relative to Bragg mode that appear simultaneously in the laser output. One way for having a single mode in AR DFB laser is by employing an equivalent phase shift at the center of the grating structure. These lasers are also named QWS-DFB and have high efficiency but are sensitive to the reflection at the facets. LCDFB lasers use a lithographic fabrication step to define the distributed feedback grating, avoiding subsequent regrowth. This makes this type of lasers not only easier to fabricate but also less expensive [2-7]. For the first time, this article reports on the stability of LC-DFB lasers against Spatial Hole Burning (SHB), maximum output power and Side Mode Suppression Ratio (SMSR) values above threshold conditions.

The theoretical analysis is carried out by simultaneously solving both the coupled wave and rate equations using the transfer matrix method. In section 2, the theory and the physical model are explained. Section 3 explains the results and the conclusion is summarized and discussed briefly in section 4.

\section{THEORETICAL AND PHYSICAL MODEL}

Figure 1 illustrates a simple design of a five-layered LCDFB laser and its features. In this figure, $d, h$ and $t$ are the active, buffer and wave guide layer thicknesses respectively.

In Figure 2, a clearer view of a LC-DFB laser is illustrated. It can be seen that there is a grating over the waveguide at both sides of the central area which in turn makes a distributed reflection in the laser.

We consider the direction of the electric field along y-axis, (Transverse electric (TE) mode). Electromagnetic waves propagate both in positive and negative directions along the Zaxis in the cavity.

The modified coupled-mode equations as formulated in [8] are:

$$
\left\{\begin{array}{c}
\frac{d A(z)}{d z}-j \delta A(z)=j \kappa B(z) \\
-\frac{d B(z)}{d z}-j \delta A(z)=j \kappa A(z)
\end{array}\right.
$$

where $A$ and $B$ are the forward and backward propagating complex amplitudes of the field respectively; $\kappa$ is the coupling coefficient between forward and backward propagating modes; $\delta=\beta-\beta_{0}$ is the Bragg wavelength detuning; $\beta_{0}=m \pi / \Lambda$ is the propagation constant, in which $m$ is the grating order.

Carrier density in the active region follows the rate equation. This equation for the single-moded DFB laser is [9]:

$$
\frac{\partial N(z, t)}{\partial t}=\frac{J}{q d}+\left[A+B N(z, t)+C N(z, t)^{2}\right]-G(z, t) S(z, t)
$$

where $N(z, t)$ and $S(z, t)$ are carrier and photon densities respectively, $J$ is the input current density; $q$ is the electronic charge; $d$ is the active layer thickness; $A, B$ and $C$ are the nonradiative, spontaneous and auger rates respectively. Photon density at every point is proportional to the sum of the square 
of forward and backward traveling wave amplitudes. In (2), $G$ is the stimulated emission rate.

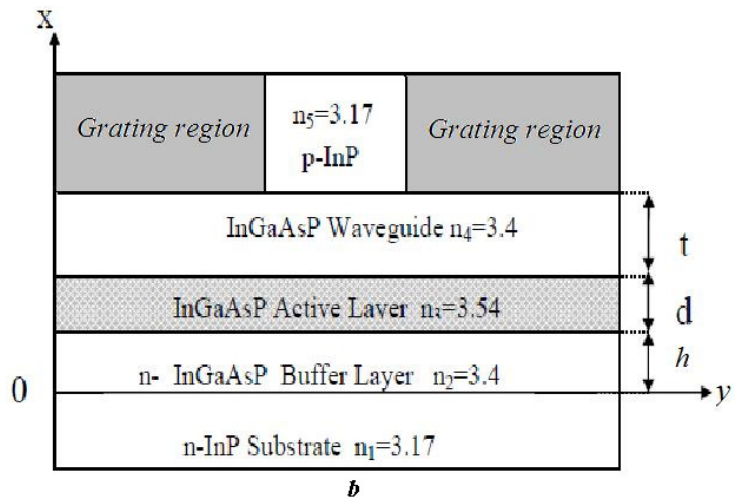

Fig. 1. Simple design of a DFB laser structure with lateral coupling

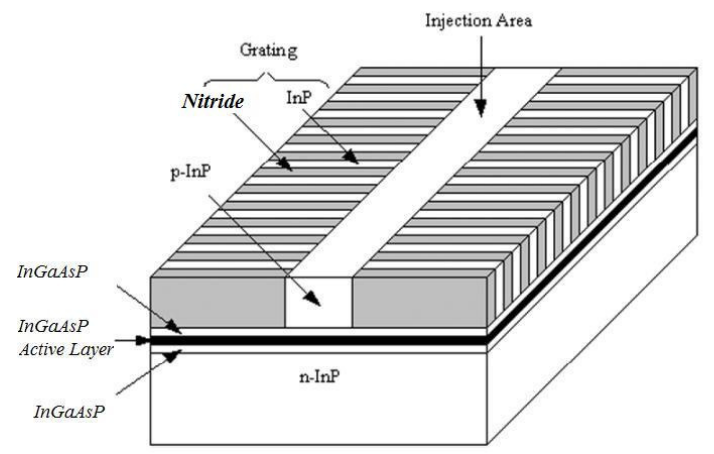

Fig. 2. The schematic of laterally-coupled distributed feedback laser [7].

Analysis is based on solving both (1) and (2) simultaneously. The method employed is based upon the transfer matrix method (TMM) $[10,11]$.

TABLE I. THE VALUES OF THE PARAMETERS

\begin{tabular}{|c|c|}
\hline Parameters & Values \\
\hline Cavity length $(\mathrm{L})$ & $500 \mu \mathrm{m}$ \\
\hline Laser wavelength $\left(\lambda_{0}\right)$ & $1.5 \mu \mathrm{m}$ \\
\hline Buffer layer thickness $(\mathrm{h})$ & $150 \mathrm{~nm}$ \\
\hline waveguide layer thickness $(\mathrm{t})$ & $150 \mathrm{~nm}$ \\
\hline $\begin{array}{c}\text { Internal dissipation of the } \\
\text { active layer }\left(\alpha_{\text {int }}\right)\end{array}$ & $20 \mathrm{~cm}^{-1}$ \\
\hline Improved line width coefficient $\left(\alpha_{0}\right)$ & 6 \\
\hline Spontaneous emission factor $(\beta)$ & $10^{-4}$ \\
\hline Nonradiative combination rate $(\mathrm{A})$ & $10^{8} \mathrm{~s}^{-1}$ \\
\hline Spontaneous recombination rates $(\mathrm{B})$ & $8 \times 10^{-11} \mathrm{~cm}^{3} \mathrm{~s}^{-1}$ \\
\hline Auger recombination rates $(\mathrm{C})$ & $7.5 \times 10^{-29} \mathrm{~cm}^{6} \mathrm{~s}^{-1}$ \\
\hline
\end{tabular}

Amplified spontaneous emission spectrum in DFB laser is computed using (3). Both Green's function and TMM are employed in the analysis [12].

$$
P_{A S E}=\frac{h c}{\lambda} \frac{\left(1-r_{2}^{2}\right)}{y_{22}+r_{1} y_{21}-r_{2} y_{12}-r_{1} r_{2} y_{11}} \int_{0}^{L} n_{s p}(z) g(z)|E(z)|^{2} d z
$$

where $r_{1}$ and $r_{2}$ are the facet reflection coefficients of the laser, $n_{s p}$ is the population inversion coefficient, $y_{i j}$ are elements in the transfer matrix and $\mathrm{E}(\mathrm{z})$ is the solution of coupled wave equations.

In this method, the cavity length is divided in to 200 equal parts and in every part different parameters of the laser including coupling coefficient, carrier density, refractive index and density of photons are assumed to be constant. But their values are different from one part to the next. Ref. [7] has been used as the bench mark for our calculations for threshold conditions to the first order grating.

The values of the parameters used in the calculations are presented in Table 1. In LC-DFB laser structure by choosing a suitable ridge height and width and also an appropriate input current, a structure with minimum flatness parameter above the threshold conditions is obtained.

For modeling purposes, first the effective refractive index method is employed and the changes both in the $X$ and $Y$ directions are calculated. Ultimately, the TMM only along the $Z$ direction is employed. The above procedure provides a simulation in all 3 directions. The effective refractive index under the ridge and the area around it has also been considered. Then the effective index calculations were used to compute the lateral coupling coefficient on both sides of the ridge.

\section{RESULTS AND DISCUSSIONS}

Figure 3 shows the Amplified Spontaneous Emission (ASE) spectrum with respect to $\delta L$, in laterally coupled distributed feedback laser structure for various ridge widths, at constant current. This figure shows various side modes and the Bragg mode (the middle mode), which is amplified. In this figure it can be seen that with constant normalized current, $b=3$, with structure having $W=2 \mu \mathrm{m}$, maximum value of the SMSR which is approximately $49 \mathrm{~dB}$, is achieved.

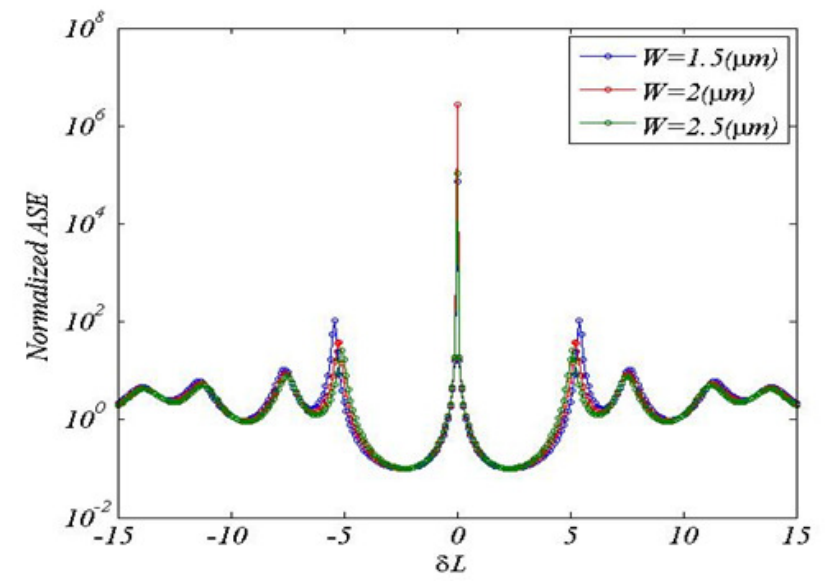

Fig. 3. Amplified Spontaneous Emission spectrum of the $\delta L$ for different ridge widths in LC-DFB structure above threshold. 
In Figure 4, we see that for the maximum value of SMSR for ridge width $W=2 \mu \mathrm{m}$ and grating amplitude $a=0.1 \mu \mathrm{m}$ with normalized current $b=2$, a value of $51 \mathrm{~dB}$ is achieved.

Figure 5 shows changes of the output power with normalized current for different values of the active region thickness. As can be seen from Figure 5, with increasing input current, output power $P_{\text {out }}$ increases and by increasing the thickness of the active region, the slope of the output power rises. With constant normalized current $b=3$, output power greater than $40 \mathrm{~mW}$ is achieved. The slope of Pout with respect to normalized current is proportional to the external quantum efficiency. By increasing $d$, light intensity and external quantum efficiency of the laser are reduced.

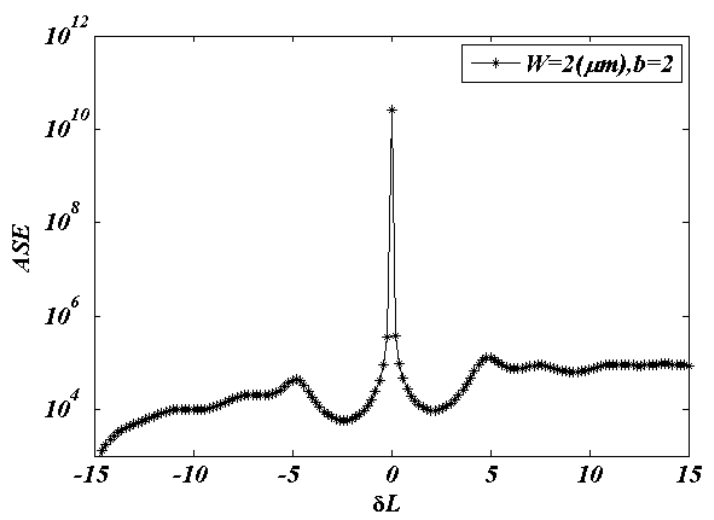

Fig. 4. Amplified Spontaneous Emission spectrum of the $\delta L$ for active region with thickness $d=1.5 \mu \mathrm{m}$ in LC-DFB structure above threshold.

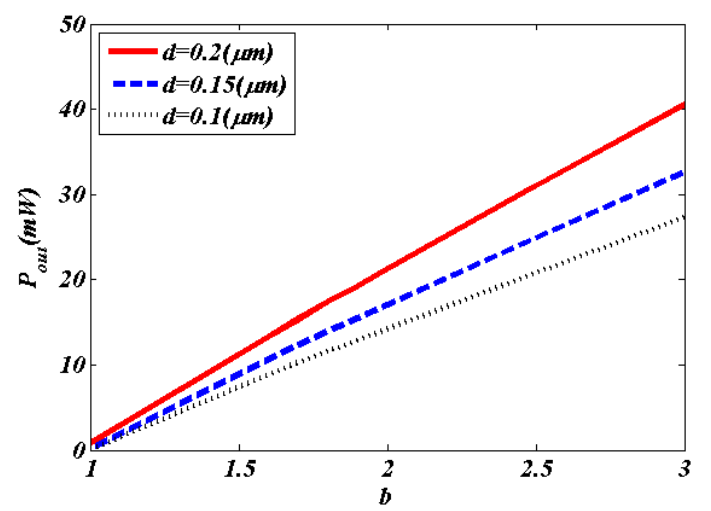

Fig. 5. Output power vs. normalized current for LC-DFB laser structure for different values of active region thickness.

Figure 6 shows the variation of Bragg deviation with different values of the active region thickness, (with respect to normalized current). Changes in the mean normalized deviation from the Bragg mode $(\delta L)$ for $d$ equal or greater than $0.2 \mu \mathrm{m}$ is almost negligible.

To better understand the effects of the electrical current on the performance of the device above the threshold, Figure 7 illustrates the changes in $\lambda$ for different values of the active region thickness. The wavelength variations and tenability of the device is estimated to be about $0.15 \mathrm{~nm}$.

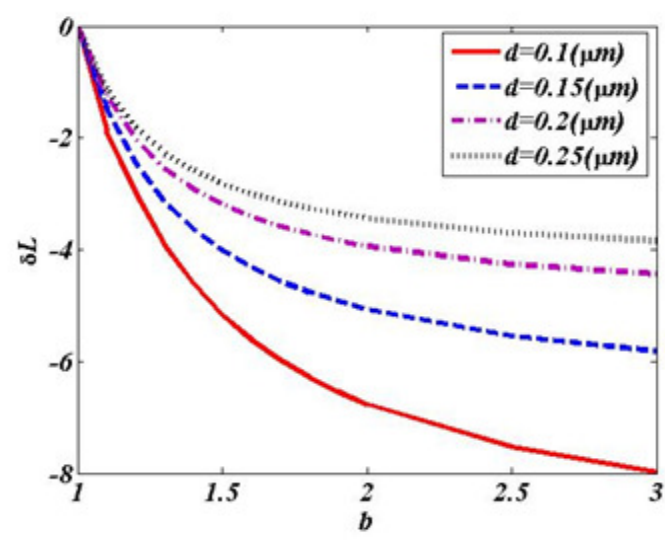

Fig. 6. Changes in the mean normalized deviation from the Bragg mode, $\delta L$ vs. normalized current for LC-DFB laser for different values of the active region thickness.

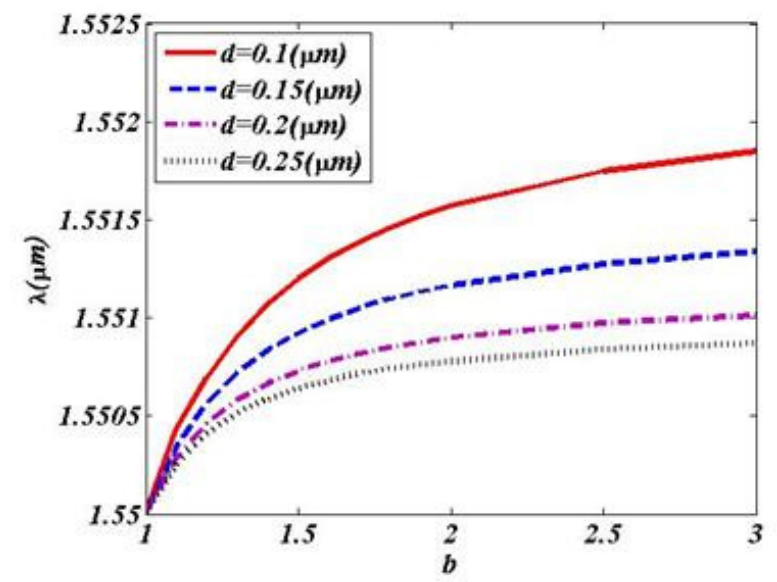

Fig. 7. Changes in the output wavelength vs. normalized current for LCDFB laser structure with different values of active region thickness.

\section{CONCLUSION}

In this paper, the LC-DFB laser above threshold condition has been analyzed and modeled for the first time. An output power more than $40 \mathrm{~mW}$ and SMSR values greater than $50 \mathrm{~dB}$ were achieved. Variations of structural parameters are considered in the simulations. The minimum changes in wavelength occurred with smaller values of the active layer thickness. The above advantages together with the device being single-moded, perhaps make this laser the most suitable choice for optical telecommunications systems.

\section{REFERENCES}

[1] K. Nosu, K. Iwashita, "A consideration of factors affecting future coherent lightwave communication", Journal of Lightwave Technol. Vol. 6, No. 5, pp.686-694, 1988

[2] R. Millett, K. Hinzer, T. Hall, H. Schriemer, "Simulation analysis of higher order laterally-coupled distributed feedback lasers", IEEE Journal of Quantum Electronics, Vol. 44, No. 12, pp. 1145-1151, 2008 
[3] R. Millett, H. Awad, M. Poirier, V. Tolstikhin, T. Hall, K. Hinzer, H. Schriemer, "Design and fabrication of a $\lambda / 4$ phase shifted $1310 \mathrm{~nm}$ laterally-coupled distributed-feedback laser", Conference on Lasers and Electro-Optics, 2008 and 2008 Conference on Quantum Electronics and Laser Science, CLEO/QELS, San Jose, 2008

[4] R Millett, K Hinzer, A Benhsaien, T. J. Hall, H Schriemer "The impact of laterally coupled grating microstructure on effective coupling coefficients", Nanotechnology, Vol. 21, No. 13, Art. No. 134015, 2010

[5] R. D. Martin, S. Forouhar, S. Keo, R. J. Lang, R. G. Hunsperger, R. Tiberio, P. F. Chapman, "InGaAs-GaAs-AIGaAs laterally-coupled distributed feedback (LC-DFB) ridge laser diode", Electronics Letters, Vol. 30 No. 13, pp. 1058-1060, 1994

[6] S. Forouhar, R.M. Briggs, C. Frez, K.J. Franz, A.Ksendzov. Highpower laterally coupled distributed-feedback GaSb-based diode lasers at $2 \mu \mathrm{m}$ wavelength" Applied Physics Letters, Vol. 100, No. 3, 031107, 2012
[7] J. Wang, J. Tian, P. Cai, B. Xiong, Ch. Sun, Y. Luo, " $1.55 \mu \mathrm{m}$ AlGaInAs-InP Laterally Coupled Distributed Feedback Laser", IEEE Photonics Technology Letters, Vol. 17, No. 7, pp. 1372 - 1374, 2005

[8] W. Streifer, D. R. Scifres, R. D. Burnham, "Coupled wave analysis of DFB and DBR lasers", IEEE Journal of Quantum Electronics, Vol. 13, No. 4, pp. 134-141, 1977

[9] G. P. Agrawal, A. K. Dutta, Semiconductor lasers, 2nd Ed., New York, Von Nostrand Reinhold, 1993

[10] F. Shahshahani, V. Ahmadi, "Analysis of relative intensity noise in tapered grating QWS-DFB laser diodes by using three rate equations model", Solid State Electronics, Vol. 52, No. 6, pp. 857-862, 2008

[11] T. Makino, "Transfer-matrix formulation of spontaneous emission noise of DFB semiconductor lasers", Journal of Lightwave Technology, Vol. 9, No. 1, pp. 84-91, 1991

[12] T. Makino, J. Glinski, "Transfer matrix analysis of the amplified spontaneous emission of DFB semiconductor laser amplifier", IEEE Journal of Quantum Electronics, Vol. 24, No. 8, pp. 1507-1518, 1988 